Pacific Journal of Mathematics

REGULAR COVERING SURFACES OF RIEMANN SURFACES 


\title{
REGULAR COVERING SURFACES OF RIEMANN SURFACES
}

\author{
SIDNEY M. HARMON
}

Introduction. The homotopy and homology groups of a given arcwise connected surface are topological invariants. A smooth covering surface $F^{*}$ is a locally-topological equivalent of its base surface $F$. Consequently, it is natural that the fundamental and homology groups of $F^{*}, T\left(F^{*}\right)$ and $H\left(F^{*}\right)$ respectively, should be related to those of $F$, $T(F)$ and $H(F)$ respectively. In this paper the term homology is always used for the 1-dimensional case. The cover transformations of a covering surface $F^{*}$ are topological self-mappings such that corresponding points have the same projection on $F$. These cover transformations form a group which we will denote by $\Gamma\left(F^{*}\right)$. The homology properties of $F$ should influence $\Gamma\left(F^{*}\right)$ by means of the composition of the self-topological mapping and the locally-topological mapping $F^{*} \rightarrow F$.

Section 1 considers the general class of smooth covering surfaces on which there exists a continuation along every arc of the base surface. We refer to such a covering surface as a regular covering surface $F^{*}$. A number of results are collected and put into the form in which they are needed to derive the main theorems. The class $\left\{F^{*}\right\}$ is shown to form a complete lattice. Next there is shown a one to one correspondence between all subgroups $N_{i} \subset T(F)$, such that $N_{i}$ contains the commutator subgroup $N_{c}$ of $T(F)$, and the set of all subgroups $H_{i} \subset H(F)$. This correspondence leads to isomorphisms which relate the associated subgroups.

Section 2 considers a special class of regular covering surfaces $\left\{F_{h}^{*}\right\}$ in which $F_{h}^{*}$ is characterized by the properties that it corresponds to a normal subgroup of $T(F)$ and $\Gamma\left(F_{h}^{*}\right)$ is Abelian. In our notation these covering surfaces form the class of homology covering surfaces (cf. Kerékjárto [5]). An equivalent characterization of the property that $F^{*}$ corresponds to a normal subgroup is the assumption that above any closed curve on $F$ there never lie two curves on $F^{*}$ one of which is closed and the other open. There are derived here for $\left\{F_{h}^{*}\right\}$ an isomorphism and correspondence theorem which relates subgroups $\Gamma_{i} \subset \Gamma\left(F_{h}^{*}\right)$ to quotient groups of $H(F)$ and $T(F)$. The class $\left\{F_{h}^{*}\right\}$ is shown to form a complete and modular lattice. If the base surface $F$ is an orientable or non-orientable closed surface, with covering surface $F_{n}^{*}$, the rank of $\Gamma\left(F_{h}^{*}\right)$ is determined in terms of the genus of $F$ and the

Received December 3, 1958. This paper contains a part of the author's doctoral thesis which was completed at the University of California at Los Angeles, together with some extensions. The author is indebted to Professor Leo Sario for his guidance in its preparation. 
rank of an associated subgroup $H_{i} \subset H(F)$.

Section 3 considers the Schottky covering surface $F_{S}^{*}$ of a closed orientable surface. We denote the boundary of the conformal equivalent of $F_{S}^{*}$ in the plane by $E_{S}$. There is obtained here a criterion for the vanishing of the linear measure of $E_{S}$.

We will refer to functions defined on a Riemann surface by an abbreviated notation as follows: Green's functions by $G$, nonconstant analytic functions with finite Dirichlet integral by $A D$ and non-constant analytic bounded functions by $A B$. We denote the class of Riemann surfaces on which there does not exist any $G, A D$ and $A B$ functions respectively by $O_{G}, O_{A D}$ and $O_{A B}$. If $W$ is an open (non-compact) Riemann surface we are led to the problem of studying it from the following comparative viewpoint (Sario [13]). Suppose that $P$ is a property of all closed (compact) Riemann surfaces, determine open Riemann surfaces which possess the same property. Recently Mori [8] established a connection between homology covering surfaces and the classes $O_{G}, O_{A D}$ and $O_{A B}$.

Section 4 applies the results of the previous three sections to the classification of Riemann surfaces. It considers regular covering surfaces of a closed Riemann surface $F$ of genus $p$. We refer to the covering surface of $F$ which corresponds to $N_{c} \subset T(F)$ as the commutator covering surface $F_{c}^{*}$. It is shown that the results obtained in [8] for homology covering surfaces $F_{h}^{*}$ with respect to $O_{A D}, O_{G}$ and $O_{A B}$ may be applied to any regular covering surface $F_{i}^{*}$ which is weaker than $F_{c}^{*}$. In the case of $O_{A B}$ this yields for $F_{i}^{*}$ a criterion in terms of the generators of quotient groups of $T(F)$ and $H(F)$. A generalization of Painlevé's problem for an open Riemann surface is proved, and there is also obtained a criterion based on vanishing linear measure of a plane point set which determines that a Schottky covering surface is in $O_{A B}$.

\section{Regular Covering Surfaces.}

1.1. Definitions. A surface is a connected Hausdorff space on which there exists an open covering by sets which are homeomorphic with open sets of the 2-dimensional Euclidean space.

A surface $F^{*}$ is a smooth covering surface of a base surface $F$ if there exists a mapping $f: F^{*} \rightarrow F$ such that for every $p^{*} \in F^{*}$ a neighborhood $V^{*}$ of $p^{*}$ is mapped topologically onto a neighborhood $V$ of $p=f\left(p^{*}\right) \in F$.

$F^{*}$ is a regular covering surface of $F$ if it is smooth and if every arc $\gamma$ on $F$ can be continued along $\gamma$ from any point over the initial point of $\gamma$. [2] (The term "unramified and unbounded"' also appears in the literature instead of the term "regular" used here.) 
1.2. Fundamental Group. The results in this subsection are needed for the later treatment and may be found or are implied in the literature; and the development closely parallels that of Ahlfors and Sario [2]. The following result is well-known.

Lemma 1. Let $\{\gamma\}$ be the homotopic classes of those curves from 0 on $F$ which have a closed continuation $\left\{\gamma^{*}\right\}$ from $0^{*} \in F^{*}$. Then $D=\{\gamma\}$ is a subgroup of the fundamental group $T(F)$ with origin at 0 .

Let the notation $\left(F^{*}, f\right)$ and $F$ represent a regular covering surface $F^{*}$ of $F$ with topological mapping $f: F^{*} \rightarrow F$ and homotopic classes originating at $0^{*}$ where $f\left(0^{*}\right)=0$. We will identify $\left(F_{1}^{*}, f_{1}\right)$ and $\left(F_{2}^{*}, f_{2}\right)$ if there exists a topological mapping $\phi: F_{1}^{*} \rightarrow F_{2}^{*}$ such that $f_{1}=f_{2} \circ \dot{\phi}$ and $\phi\left(0_{1}^{*}\right)=0_{2}^{*}$. It is clear that this identification is defined by means of an equivalence relation.

The proofs of the following proposition and of the subsequent Lemmas 2 through 4 may be obtained from reference [2] or [9].

Proposition 1. The mapping $\phi$ in the identification of $\left(F_{1}^{*}, f_{1}\right)$ and $\left(F_{2}^{*}, f_{2}\right)$ with $\phi\left(0_{1}^{*}\right)=0_{2}^{*}$ is uniquely determined.

With the foregoing identification, we obtain

LEMMA 2. There exists a one to one correspondence between identified pairs $\left(F^{*}, f\right)$ and the subgroups $D$ of $T(F)$. Two pairs can be represented by means of the same $\left(F^{*}, f\right)$ if and only if the corresponding subgroups are conjugate.

Lemma 3. The fundamental group $T\left(F^{*}\right)$ of $\left(F^{*}, f\right)$ is isomorphic with the corresponding subgroup $D$ of $T(F)$.

If $\left(F_{2}^{*}, f\right)$ covers $F_{1}^{*}$ and $\left(F_{1}^{*}, f_{1}\right)$ covers $F$, then it is clear that $\left(F_{2}^{*}, f_{1} \circ f\right)$ covers $F$ where $f_{1} \circ f\left(0_{2}^{*}\right)=0$. If two pairs $\left(F_{2}^{*}, f_{2}\right)$ and $\left(F_{1}^{*}, f_{1}\right)$ cover $F$, we say that the former is stronger than the latter if and only if there exists an $f$ such that $\left(F_{2}^{*}, f\right)$ covers $F_{1}^{*}$ and $f_{2}=f_{1} \circ f$. This relation is clearly transitive.

Let $D_{1}$ and $D_{2}$ be the subgroups of $T(F)$ which correspond respectively to $\left(F_{1}^{*}, f_{1}\right)$ and $\left(F_{2}^{*}, f_{2}\right)$, then we have

LEMma 4. The pair $\left(F_{2}^{*}, f_{2}\right)$ is stronger than $\left(F_{1}^{*}, f_{1}\right)$ if and only if $D_{2} \subset D_{1}$.

1.3. Complete Lattice Theorem. By means of Lemmas 2 and 4, we obtain an ordering of the regular covering surfaces according to relative strength which is isomorphic with the ordering of the corresponding subgroups of $T(F)$ by inclusion.

Let $\left\{D_{a}\right\}$ with a in the index set $A$ be a finite or infinite subset of 
a lattice $L$. Then $L$ is complete if for all $\left\{D_{a}\right\} \subset L$, there exists in $L$ a least upper bound $\bigcup_{a \in A} D_{a}$ and a greatest lower bound $\bigcap_{a \in A} D_{a}$.

THEOREM 1. The system of regular covering surfaces of $F$ is a complete lattice.

Proof. The system of subgroups $\left\{D_{a}\right\}$ of $T(F)$ with $a \in A$ is partially ordered by inclusion. Also the union of any number of subgroups $\left\{D_{a_{i}}\right\}$ for $a_{i} \in A$ is a subgroup $\bigcup_{a_{i} \in A} D_{a_{i}}$ which is the least upper bound for $\left\{D_{a_{i}}\right\}$. Similarly, the intersection of any number of subgroups $\left\{D_{a_{i}}\right\}$ is a subgroup $\bigcup_{a_{i} \in A} D_{a_{i}}$ which is the greatest lower bound for $\left\{D_{a_{i}}\right\}$. Consequently the system of subgroups $\left\{D_{a}\right\}$ is a complete lattice. Because of the isomorphy obtained from Lemmas 2 and 4 , the corresponding regular covering surfaces form a complete lattice.

It can be shown that any complete lattice has a zero and a universal element. The weakest covering surface of $F$ corresponds to $T(F)$ and is $F$ itself or $\left(F^{*}, e\right)$, where $e$ is the identity; the strongest covering surface corresponds to the unit element of $T(F)$ and is the universal covering surface of $F$.

\subsection{Relations Between Fundamental and Homology Groups.} The commutator subgroup of $T(F)$ will be denoted by $N_{c}$. The covering surface $F_{c}^{*}$ which corresponds to $N_{c}$ will be referred to as the commutator covering surface. (Uberlagerungsfläche der Integralflunktionen, Weyl [17])

Lemma 5. (Nevanlinna [9; 61-63]) There exists a homomorphism from the elements of $T(F)$ onto the elements of $H(F)$ for which the kernel is the commutator subgroup.

If $\theta$ is a homomorphism from $T$ to $H$ with kernel $K$, the fundamental theorem for group homomorphisms yields the isomorphism $T / K \cong H$. A second fundamental theorem for group homomorphisms may be stated in the following form (Kurosh [6]).

Lemma 6. Let $\theta: F \rightarrow H$ be a homomorphism with kernel $K$. Then

(i) There is a one to one correspondence between subgroups $N_{i}$ of $T$ such that $T \supset N_{i} \supset K$ and all subgroups $H_{i}$ of $H$. In this correspondence $H_{i}$ consists of all images of elements of $N_{i}$ and $N_{i}$ consists of all inverse images of elements of $H_{i}$.

(ii) If $N_{i}$ is normal in $T$ then $H_{i}$ is normal in $H$ and conversely.

(iii) If $N_{i}$ and $K$ are normal in $T$ then $T / N_{i} \cong(T / K) /\left(N_{i} / K\right)$.

THEOREM 2. Let $\left\{N_{i}\right\}$ be the set of all subgroups such that $T(F) \supset$ $N_{i} \supset N_{c}$ and let $\left\{H_{i}\right\}$ be the set of all subgroups $H_{i} \subset H(F)$. Then 
(i) There exists a one to one correspondence between $\left\{N_{i}\right\}$ and $\left\{H_{i}\right\}$. In this correspondence $H_{i}$ consists of all images of elements of $N_{i}$ and $N_{i}$ consists of all inverse images of elements of $H_{i}$.

(ii) $N_{i} / N_{c} \cong H_{i}$.

Proof. To prove the first part, we use the homomorphism of Lemma 5 $\theta: T(F) \rightarrow H(F)$ with kernal $N_{c}$. Part (i) of the theorem is then an immediate consequence of Lemma 6 (i).

To obtain the isomorphism (ii) we note that $N_{c}$ is normal in $N_{i}$ and that the restricted homomorphism $\theta: N_{i} \rightarrow H_{i}$ is onto. We apply the fundamental theorem for group homomorphisms which yields the required isomorphism.

If in Theorem 2 we set $N_{i}=T(F)$, we obtain $T(F) / N_{c} \cong H(F)$ as a special case.

1.5. Relations Between the Fundamental Group and the Group of Cover Transformations.

DeFinition. A cover transformation of a regular covering surface $\left(F^{*}, f\right)$ is a topological self-mapping $\phi$ such that, for every $p^{*} \in F^{*}$, $\phi\left(p^{*}\right)$ and $p^{*}$ have the same projection.

The totality of cover transformations on $F^{*}$ clearly form a group. We will denote this group by $\Gamma\left(F^{*}\right)$.

In the sequel, unless otherwise indicated, $D$ or $D_{i}$ will refer to the subgroup of $T(F)$ which corresponds to the covering surface $F^{*}$ or $F_{i}^{*}$ respectively, according to the specifications of Lemma 2 . We note that $\Gamma\left(F^{*}\right)$ and the normalizer of $D$ are unaffected by the choice of 0 and $0^{*}$.

Lemma 7. [9;83] Let $M$ be the normalizer of $D$ in $T(F)$. Then there exists a homomorphism $\phi: M \rightarrow \Gamma\left(F^{*}\right)$ with the kernel $D$.

THEOREM 3. Let $\left\{D_{i}\right\}$ be the set of all subgroups $D_{i}$ such that $M \supset D_{i} \supset D$ and let $\left\{\Gamma_{i}\right\}$ be the set of all subgroups of $\Gamma\left(F^{*}\right)$. Then

(i) There exists a one to one correspondence between $\left\{D_{i}\right\}$ and $\left\{\Gamma_{i}\right\}$. In this correspondence $\Gamma_{i}$ consists of all images of elements of $D_{i}$, and $D_{i}$ consists of all inverse images of elements of $\Gamma_{i}$.

(ii) $\Gamma_{i} \cong D_{i} / D$.

Proof. We use the homomorphism $\phi$ of Lemma 7 with kernel $D$. Part (i) of the theorem is then an immediate consequence of Lemma 6 (i). To obtain the isomorphism (ii), we note that $D$ is the kernel of $\phi$ and $D$ is normal in $M$ and, therefore, normal in $D_{i} \subset M$. By (i), $\phi$ maps $D_{i}$ onto $\Gamma_{i}$. The restriction of $\phi$ to $D_{i}$ in conjunction with the fundamental theorem for group homomorphisms yields the required isomorphism. 
If in Theorem 3 we set $D_{i}=M$, we find from part (i) of the theorem that $M$ is mapped onto $\Gamma\left(F^{*}\right)$. Consequently, we obtain from (ii),

$$
\Gamma\left(F^{*}\right) \cong M / D,
$$

as a special case.

CoRollary. If $D$ is normal in $T(F)$, then the one to one correspondence and isomorphism specified in Theorem 3 holds for all subgroups $D_{i}$, such that $T(F) \supset D_{i} \supset D$.

Proof. If $D$ is normal in $T(F)$, then the normalizer of $D$ is $T(F)$. We replace $M$ in Theorem 3 by $T(F)$ and obtain the required result.

A special case of the corollary is obtained if in Theorem 3 (ii) we set $D_{i}=T(F)$. We then find that

$$
\Gamma\left(F^{*}\right) \cong T(F) / D .
$$

\section{Homology Covering Surfaces.}

2.1. Definitions And BASic Result. A regular covering surface of $F$ is normal if it corresponds to a normal subgroup of $T(F)$ [2]. (The term "unramified, unbounded and regular" also appears in the literature instead of the single term "normal" used here.)

Proposition 2. (Seifert-Threlfall $[16 ; 196])$ If $\left(F^{*}, f\right)$ is a normal covering surface of $F$, then there exists a unique cover transformation which carries any given point $p^{*} \in\left(F^{*}, f\right)$ into a prescribed point $p_{1}^{*}$ with the same projection.

A regular covering surface is referred to as a commutative covering surface if its group of cover transformations is Abelian.

A homology covering surface is a covering surface which is simultaneously normal and commutative.

\subsection{Criterion Theorem.}

THEOREM 4. A regular covering surface $F_{i}^{*}$ is a homology covering surface of $F$ if and only if it is weaker than the commutator covering surface $F_{c}^{*}$, or equivalently, if and only if $N_{i} \supset N_{c}$, where $F_{i}^{*}$ and $F_{c}^{*}$ correspond respectively to the subgroups $N_{i}$ and $N_{c}$ of $T(F)$.

Proof. To prove the sufficiency of the condition, we first consider $F_{c}^{*}$ which corresponds to $N_{c}$ which is clearly normal in $T(F)$. By the isomorphism (2), we obtain $\Gamma\left(F_{c}^{*}\right) \cong T(F) / N_{c}$. The latter quotient group is Abelian; for if $a, b \in T(F), a b(b a)^{-1}=a b a^{-1} b^{-1} \in N_{c}$; hence $N_{c} a b=N_{c} b a$. 
By hypothesis, $F_{i}^{*}$ is weaker than $F_{c}^{*}$; consequently by Lemma 4, $N_{i} \supset N_{c}$. From the fact that $T(F) / N_{c}$ is Abelian and $N_{i} \supset N_{c}$ in conjunction with Lemma 6 (ii), it follows that any subgroup $N_{i}$ which contains $N_{c}$ is normal. We conclude from Lemma 6 (iii) that $T(F) / N_{i}$ is Abelian. The latter quotient group is isomorphic to $\Gamma\left(F_{i}^{*}\right)$ by the special case (2). We conclude that $F_{i}^{*}$ is simultaneously a normal and commutative covering surface and therefore a homology covering surface.

Conversely, we suppose that $F_{i}^{*}$ is a homology covering surface. From the special case (2), we obtain $\Gamma\left(F_{i}^{*}\right) \cong T(F) / N_{i}$. By hypothesis, the left member of the isomorphism is Abelian; consequently $T(F) / N_{i}$ is Abelian. Because of the commutativity of $T(F) / N_{i}$ and the normality of $N_{i}$, we obtain for $a, b \in T(F), N_{i} a b a^{-1} b^{-1}=N_{i}$; therefore, $N_{i} \supset N_{c}$. We conclude, by Lemma 4 , that $F_{i}^{*}$ is weaker than $F_{c}^{*}$.

The last statement of the theorem is an immediate consequence of Lemma 4.

\subsection{ISOMORPHISM AND CORRESPONDENCE THEOREM.}

THEOREM 5. Let $\left\{F_{h i}^{*}\right\}$ be the set of all homology covering surfaces of $F$ under the identification of Lemma 2, and let $\left\{N_{h i}\right\}$ be the set of all corresponding subgroups of $T(F)$ under the isomorphy of Lemma 3; such that $T\left(F_{h i}^{*}\right) \cong N_{h i}$. Let $\left\{H_{i}\right\}$ be the set of all subgroups of $H(F)$ under the correspondence indicated in Theorem 2, such that $N_{h i} / N_{c} \cong H_{i}$. Then

(i) $\Gamma\left(F_{h i}^{*}\right) \cong H(F) / H_{i} \cong T(F) / N_{h i} \cong\left[T(F) / N_{c}\right] /\left(N_{h i} / N_{c}\right)$.

(ii) There exists a one-to-one correspondence between the identified sets $\left\{F_{h i}^{*}\right\}$ and the sets $\left\{N_{h i}\right\}$ and $\left\{H_{i}\right\}$.

Proof. To derive the first and second isomorphisms of (i), we note that because of the commutativity of the homology groups, $H_{i}$ is normal in $H(F)$. We consider the composite mapping $\phi \circ \theta$,

$$
\begin{aligned}
& \phi \circ \theta[T(F)]=\phi[H(F)]=H(F) / H_{i}, \\
& \phi \circ \theta[a \in T(F)]=\phi\left(a^{\prime}\right)=H_{i} a^{\prime} .
\end{aligned}
$$

This mapping is composed of the homomorphism $\theta$ of Lemma 5 and the natural homomorphism $\phi$; consequently the composition is a homomorphism. The kernel of $\phi \circ \theta$ consists of all $a \in T(F)$ such that $H_{i} a^{\prime}=H_{i}$. We note that by Theorem $4, N_{h i} \supset N_{c}$; hence Theorem 2 (i) is applicable. From the specifications in Theorem 2 (i) for $\theta: N_{h i} \rightarrow H_{i}$, we find that the kernel of $\phi \circ \theta$ is precisely $N_{h i}$. The fundamental theorem of group homomorphism, together with the special case (2), now yield

$$
\Gamma\left(F_{i}^{*}\right) \cong T(F) / N_{h i} \cong H(F) / H_{i}
$$


To derive the third isomorphism of (i), we note that $N_{h i}$ and $N_{c}$ are normal in $T(F)$. Hence an application of the fundamental theorem for group homomorphisms yields the result.

For the proof of (ii), we note that by Theorem 4, any homology covering surface $F_{h i}^{*}$ satisfies $N_{h i} \supset N_{c}$. Hence Theorem 2 is applicable. We apply Theorem 2 (i) to obtain a one-to-one correspondence between $\left\{N_{h i}\right\}$ and $\left\{H_{i}\right\}$. The one-to-one correspondence obtained is carried through $\left\{N_{h i}\right\}$ to $\left\{F_{h i}^{*}\right\}$, under the postulated identification, by means of Lemma 2. This completes the proof of the theorem.

2.4. Complete and Modular Lattice Theorem. A lattice is called modular (Dedekind structure) if it satisfies the following weak form of the distributive law:

$$
\text { If } a \supset b \text {, then } a \cap(b \cup c)=(a \cap b) \cup(a \cap c) \text {. }
$$

LEMMA 8. (Kurosh [7]). The lattice of normal subgroups of any group is modular.

THeOREM 6. The system of homology covering surfaces $\left\{F_{h i}^{*}\right\}$ of $F$ is a complete and modular lattice.

Proof. Let $\left\{N_{h i}\right\}$ correspond to the collection $\left\{F_{h i}^{*}\right\}$. In the course of the proof of Theorem 1, it was shown that the system af subgroups $\left\{D_{i}\right\}$ of $T(F)$ is a complete lattice. $\left\{N_{h i}\right\}$ is therefore a subset of a complete lattice. From the definition of a homology covering surface and from Theorem 4 every $N_{h i}$ is normal in $T(F)$ and $N_{h i} \supset N_{c}$. The union or intersection of any number of normal subgroups of $\left\{N_{h i}\right\}$ is a normal subgroup containing $N_{c}$. Consequently, $\left\{N_{h i}\right\}$ is a sublattice and a complete lattice. By the normality of $N_{h i}$ and Lemma $8,\left\{N_{h i}\right\}$ is also a modular lattice. We conclude from Theorem 5 (ii) and Lemma 4 that $\left\{F_{h i}^{*}\right\}$ is a complete and modular lattice.

2.5. RANK OF the Group of Cover Transformations. We consider the rank of the group of cover transformations for homology covering surfaces for which the base surface $F$ is closed. In this case, $T(F)$ and $H(F)$ are finitely generated. We have

Lemma 9. (Seifert-Threlfall $[16 ; 145])$. Let $F$ be a closed surface of genus $p$. If $F$ is orientable, $H(F)$ is a free Abelian group of $2 p$ generators; if $F$ is nonorientable, $H(F)$ is the direct product of a free Abelian group of $p-1$ generators and a group of order 2.

Because the homology group of a closed surface is finitely generated. it always has a finite rank.

The following lemma is fundamental in the theory of Abelian groups. 
Lemma 10. Let $H$ be an Abelian group of finite rank $r$, and let $H_{i}$ be a subgroup of $H$. Then $H_{i}$ and $H / H_{i}$ are also of finite rank and $r(H)=r\left(H_{i}\right)+r\left(H / H_{i}\right)$.

THeOREm 7. Let $F$ be a closed surface of genus $p$, and let $\left\{F_{n i}^{*}\right\}$ be the class of homology covering surfaces of $F$ such that

$$
T\left(F_{h i}^{*}\right) \cong N_{h i} \subset T(F), \quad N_{h i} / N_{c} \cong H_{i} \subset H(F) .
$$

If $F$ is orientable, then

$$
r\left[\Gamma\left(F_{h i}^{*}\right)\right]=2 p-r\left(H_{i}\right)
$$

and

$$
0 \leqq r\left[\Gamma\left(F_{h i}^{*}\right)\right] \leqq 2 p
$$

If $F$ is nonorientable, then

$$
r\left[\Gamma\left(F_{h i}^{*}\right)\right]=p-1-r\left(H_{i}\right)
$$

and

$$
0 \leqq r\left[\Gamma\left(F_{h i}^{*}\right)\right] \leqq p-1 .
$$

In either case, $r\left[\Gamma\left(F_{h i}^{*}\right)\right]$ assumes all integral values in the indicated ranges.

Proof. We note that the rank of a free Abelian group is equal to the number of its generators, that the rank of an Abelian group in which all elements have finite order is zero, and that the rank of an Abelian group equals the sum of the ranks of the factors in the direct product decomposition of the group. Consequently, it follows from Lemma 9 , that if $F$ is orientable, $r[H(F)]=2 p$, and that if $F$ is nonorientable, $r[H(F)]=p-1$. By use of Theorem 5 (i) and Lemma 10, and by substituting for $r[H(F)]$ the values just deduced we find that if $F$ is orientable

$$
r\left[\Gamma\left(F_{h i}^{*}\right)\right]=2 p-r\left(H_{i}\right),
$$

and that if $F$ is nonorientable,

$$
r\left[\Gamma\left(F_{h i}^{*}\right)\right]=p-1-r\left(H_{i}\right) .
$$

Because $H_{i}$ is a subgroup of $H(F)$

$$
0 \leqq r\left(H_{i}\right) \leqq r[H(F)] \text {. }
$$

For each integer $n$ such that $0 \leqq n \leqq r[H(F)]$, there exists a subgroup $H_{i}$ which is generated by $n$ linearly independent elements; therefore $r\left(H_{i}\right)=n$. We conclude that if $F$ is orientable, 


$$
0 \leqq r\left[\Gamma\left(F_{n i}^{*}\right)\right] \leqq r[H(F)]=2 p,
$$

and that if $F$ is nonorientable,

$$
0 \leqq r\left[\Gamma\left(F_{h i}^{*}\right)\right] \leqq r[H(F)]=p-1 .
$$

In both cases $r\left[\left(F_{n i}^{*}\right)\right]$ assumes all integral values in the indicated ranges.

In connection with Theorem 7 it is of interest to note that the quantities $2 p$ and $p-1$ are the 1-dimensional Betti numbers for a closed orientable and a closed nonorientable surface respectively.

\section{Schottky Covering Surface of a Riemann Surface.}

3.1. Definitions for Riemann Surfaces. We shall define a Riemann surface topologically as a Hausdorff space with certain restrictive properties.

Definition. A Riemann surface $F$ is a surface together with a collection of local homeomorphisms $\{h\}$ from open sets of $F$ onto open sets of the complex plane which satisfy the following conditions.

(i) The totality of domains of $\{h\}$ form a covering of $F$.

(ii) The images of every nonnull common domain of $h_{i}$ and $h_{j} \in\{h\}$ are directly conformally equivalent in the complex plane through the composite homeomorphism $h_{i} \circ h_{j}^{-1}$.

We denote the domain of $h_{i} \in\{h\}$ by $\Delta_{i}$. If $p \in \Delta_{i}$, then $z=h_{i}(p)$ is uniquely determined. Because of condition (ii), the conformally invariant properties of $F$ are independent of the choice of $h_{i} \in\{h\}$. Consequently in considering such properties we may regard $z$ in the complex plane as a local variable instead of $p \in F$. In this paper we shall be concerned exclusively with conformally invariant properties of $F$; therefore we will resort to the local variable notation $z$ whenever it is convenient.

Definition. A complex-valued function $f$ is analytic on $F$ if and only if $f \circ h_{i}^{-1}$ is analytic on $h_{i}\left(A_{i}\right)$ for every $h_{i} \in\{h\}$ with domain $\Delta_{i}$.

Definition. A real-valued function $u$ is harmonic on $F$ if $u \circ h_{i}^{-1}$ is harmonic on $h_{i}\left(A_{i}\right)$ for every $h_{i} \in\{h\}$ with domain $\Delta_{i}$.

The Riemann surface as defined here is an orientable surface because the composite mapping $h_{i} \circ h_{j}^{-1}$ is directly conformal and consequently sense-preserving. It can be shown that the Riemann surface is topologically a countable space.

3.2. Basic Considerations. In this section the base surface $F$ is assumed to be a closed Riemann surface of finite genus $p$. By suitably 
cutting $F$, we can obtain a planar region $F_{0 S}^{\prime}$, such that an infinite number of copies of $F_{o S}^{\prime}$, when put together under special identifications of their boundaries, will generate the Schottky covering surface $F_{S}^{*}$ of $F$. The surface $F_{S}^{*}$ is a planar, open Riemann surface. We will study the boundary of the conformal equivalent of $F_{S}^{*}$ in the complex plane by means of a Schottky group.

3.3. Generators of Schottky Group. The conformal equivalent of the initial copy $F_{0 S}^{\prime}$ is an infinite region $R_{0}$, where $R_{0}$ is bounded by $2 p$ disjoint circles $Q_{i}, Q_{i}^{\prime}(i=1,2, \cdots, p)$, lying in the finite plane. We will refer to this set of circles which bound $R_{0}$ as $\left\{Q_{0}\right\}$. The $p$ pairs of circles $\left\{Q_{0}\right\}$ correspond to a system of $p$ hyperbolic or loxodromic linear transformations which generate a group of linear transformations $G$ called the Schottky group (Schottky [15]). The group $G$ can be shown to be denumerably infinite and is properly discontinuous up to a set of discrete points $E_{S}$, called the singular set of the Schottky group. The transforms of $R_{0}$ converge for $p>1$ to a nondenumerable discrete set of points $E_{S}$ which is the boundary of the conformal image of $F_{S}^{*}$ in the plane.

A set has zero linear measure if it can be covered by a sequence of disks $\left\{K_{i}\right\}$ with radii $\left\{r_{i}\right\}$ such that $\sum r_{i}$ is arbitrarily small. We will denote the linear measure of the singular set of the Schottky group by $m\left(E_{S}\right)$.

We consider a configuration of the bounding circles $\left\{Q_{0}\right\}$ corresponding to a Schottky group $G$, in order to obtain a criterion for the vanishing of $m\left(E_{S}\right)$.

Let the $2 p$. circles $\left\{Q_{0}\right\}$ be paired in such a manner that a set of $p$ hyperbolic or loxodromic linear transformations $S_{1}, \cdots, S_{p}$ operate on the extended complex plane and yield

$$
S_{1} Q_{1}=Q_{1}^{\prime}, \quad S_{2} Q_{2}=Q_{2}^{\prime}, \quad \cdots, \quad S_{p} Q_{p}=Q_{p}^{\prime},
$$

with the exterior of each $Q_{i}$ mapped into the interior of $Q_{i}^{\prime}$. The set of such generators will be designated as $\left\{S_{0}\right\}$. A general form for the transformation $S_{i}$ is

$$
S_{i}=\frac{a z+b}{c z+d}
$$

$S_{i}$ and other linear transformations in the sequel will be normalized by the condition $a d-b c=1$. The circles $\left\{Q_{0}\right\}$ have the general equations

$$
Q_{i}:\left|z-q_{i}\right|=r_{i} ; \quad Q_{i}^{\prime}:\left|z-q_{i}^{\prime}\right|=r_{i}^{\prime} .
$$

A general normalized transformation of $\left\{S_{0}\right\}$ corresponding to the circles (5) may be written as 


$$
S_{i}(z)=\frac{\frac{q_{i}^{\prime} z}{\sqrt{r_{i} r_{i}^{\prime}}}-\frac{q_{i} q_{i}^{\prime}+r_{i} r_{i}^{\prime}}{\sqrt{r_{i} r_{i}^{\prime}}}}{\frac{z}{\sqrt{r_{i} r_{i}^{\prime}}}-\frac{q_{i}}{\sqrt{r_{i} r_{i}^{\prime}}}}
$$

in which $q_{i}+r_{i} e^{i \theta}$ transforms into $q_{i}^{\prime}-r_{i}^{\prime} e^{i(2 \pi-\theta)}$.

The set $\left\{S_{0}\right\}$ corresponding to the form (6) will generate a Schottky group.

Let $\xi_{1}$ and $\xi_{2}$ denote the fixed points of a generator $S_{i}$, where $\xi_{1}$ and $\xi_{2}$ are finite. Then

$$
\xi_{1}, \xi_{2}=\frac{a-d \pm \sqrt{(a+d)^{2}-4}}{2 c} .
$$

Since $S_{i}(\infty)=q_{i}^{\prime}, S_{i}(z)=z^{\prime}$ may be expressed in terms of a cross-ratio as

$$
\frac{z^{\prime}-\xi_{1}}{z^{\prime}-\xi_{2}}=K \frac{z-\xi_{1}}{z-\xi_{2}}
$$

where $K$ is a multiplier such that

$$
K=\frac{q_{i}^{\prime}-\xi_{1}}{q_{i}^{\prime}-\xi_{2}}
$$

By simplification, this reduces to

$$
K=\left(\frac{q_{i}-q_{i}^{\prime}+\sqrt{\left(q_{i}^{\prime}-q_{i}\right)^{2}-4 r_{i} r_{i}^{\prime}}}{2 \sqrt{r_{i} r_{i}^{\prime}}}\right)^{2}
$$

We note that $K$ is independent of $z$ and that the fixed points are independent of the power of $z$. Consequently, $S_{i}^{n}(z)=z^{(n)}$ may be expressed as

$$
\frac{z^{(n)}-\xi_{1}}{z^{(n)}-\xi_{2}}=K^{n} \frac{z-\xi_{1}}{z-\xi_{2}}
$$

This yields

$$
S_{i}^{n}(z)=\frac{\left(K^{n} \xi_{2}-\xi_{1}\right) z-\xi_{1} \xi_{2}\left(K^{n}-1\right)}{\left(K^{n}-1\right) z+\xi_{2}-K^{n} \xi_{1}} .
$$

To normalize $S_{i}^{(n)}(z)$ we divide through by

$$
K^{n / 2}\left(\xi_{1}-\xi_{2}\right)=D^{\prime}
$$

and obtain

$$
S_{i}^{n}(z)=\frac{\left(\frac{K^{n} \xi_{2}-\xi_{1}}{D^{\prime}}\right) z-\frac{\xi_{1} \xi_{2}\left(K^{n}-1\right)}{D^{\prime}}}{\frac{\left(K^{n}-1\right) z}{D^{\prime}}+\frac{\xi_{2}-K^{n} \xi_{1}}{D^{\prime}}} .
$$


3.4. Isometric Circles. Because of the mappings $S_{i} Q_{i}=Q_{i}^{\prime}$ in the generation of the Schottky group, there is a particular convenience in utilizing the concept of isometric circle under a linear transformation (e.g., Ford [4]).

Let $S$ be a linear transformation expressed in the general form (4). Then length and area are unaltered in magnitude in the neighborhood of a point $z$ if and only if $|c z+d|=1$. The locus of such points for $c \neq 0$ may be written as the circle $|z+d / c|=1 /|c|$, with center $-d / c$ and radius $1 /|c|$.

DEFINITION. Let $S$ be the linear transformation

$$
S(z)=(a z+b) /(c z+d) .
$$

Then the circle

$$
I:|c z+d|=1,
$$$$
c \neq 0 \text {, }
$$

which is the complete locus of points in the neighborhood of which length and area are unaltered in magnitude by $S$ is called the isometric circle of $S$.

LEMmA 11. Let the linear transformations $S$ have $I$ as its isometric circle, and let $S(I)=I^{\prime}$. Then $S^{-1}$ has $I^{\prime}$ as its isometric circle.

Proof. By definition $S$ carries $I$ into a circle $I^{\prime}$ without alteration of lengths in the neighborhood of any point of $I$. Consequently $S^{-1}$ carries $I^{\prime}=S(I)$ back to $I$ without alteration of lengths. By the uniqueness of $I^{\prime}$, we conclude that $I^{\prime}$ is the isometric circle of $S^{-1}$.

Lemma 12. (Ford [4]). Let $I$ and $I^{\prime}$ be the isometric circles of $S$ and $S^{-1}$ respectively and let $L$ be the perpendicular bisector of the line joining the centers of $I$ and $I^{\prime}$. If $S$ is a hyperbolic, elliptic or parabolic linear transformation, $S$ is equivalent to the composition of an inversion in I followed by a reflection in the line $L$; if $S$ is loxodromic, there is in addition a rotation about the center of $I^{\prime}$ through the angle $-2 \arg (a+d)$.

THEOREM 8. Let $S$ be a linear transformation. Suppose that $S$ and $S^{-1}$ have the isometric circles $I$ and $I^{\prime}$ respectively. Then for every $n$

(i) The circles $S^{-n}(I)$ and $S^{n}\left(I^{\prime}\right)$ are equal in magnitude and $S^{-n}(I) \subset I, S^{n}\left(I^{\prime}\right) \subset I^{\prime}$.

(ii) $S^{-n}(I)$ is the isometric circle of $S^{2 n+1}$.

(iii) The radii of the circles $S^{-n}(I)$ and $S^{n}\left(I^{\prime}\right)$ are each equal to 
$1 /|c|$, where $c$ is the coefficient in the general expression for a linear transformation corresponding to $S^{2 n+1}$.

Proof. Because $S$ and $S^{-1}$ have the respective isometric circles $I$ and $I^{\prime}$, we conclude from Lemma 11 that $S(I)=I^{\prime}$. Let $L$ be the perpendicular bisector of the line joining the centers of $I$ and $I^{\prime}$. We first consider the case where $S$ is nonloxodromic. Then by Lemma 12, $S^{-n}(I)$ is obtained by successive compositions of an inversion in $I^{\prime}$ followed by a reflection in $L$, and $S^{n}\left(I^{\prime}\right)$ is obtained by successive compositions of an inversion in $I$ followed by a reflection in $L$. We note that for all linear transformations the size of the circle is influenced only by the inversion. The circles $S^{-k}(I)$ and $S^{k}\left(I^{\prime}\right)$ are symmetrical with respect to $L$ for all $k<n$. Because of the symmetry of the inversion with respect to the equal circles $I^{\prime}$ and $I$, we conclude that $S^{-n}(I)$ and $S^{n}\left(I^{\prime}\right)$ are equal. Further, from the geometrical interpretation of $S^{n}$ and $S^{-n}$ as expressed by Lemma 12, it follows that $S^{-n}(I) \subset I$ and $S^{n}\left(I^{\prime}\right) \subset I^{\prime}$.

If $S$ is loxodromic, there is in addition, in the foregoing compositions a rotation. For $S^{-k}(I)$ and $k<n$ the required rotation is $-2 k \arg [-(\alpha+d)]=-2 k \pi-2 k \arg (a+d)$ about the center of $I$, and for $S^{k}\left(I^{\prime}\right)$ the required rotation is $-2 k \arg (a+d)$ about the center of $I^{\prime}$. The circles $S^{-k}(I)$ and $S^{k}\left(I^{\prime}\right)$ are therefore symmetrical with respect to the intersection of $L$ and the line joining the centers of $I^{\prime}$ and $I$. This symmetry yields equal circles in the successive inversions with respect to the circles $I^{\prime}$ and $I$. We conclude again that $S^{-n}(I)$ and $S^{n}(I)$ are equal and that $S^{-n}(I) \subset I$ and $S^{n}\left(I^{\prime}\right) \subset I^{\prime}$. This completes the proof of part (i).

To prove part (ii) we consider $S^{2 n+1} \circ S^{-n}(I)$. The first $n$ operations by $S$ transform $S^{-n}(I)$ to $I$. The inversions associated with these transformation are all in $I$ and are of the type $S^{-(n-j)}(I)$ inverts to $S^{n-j-1}\left(I^{\prime}\right)$, where $j=0,1, \cdots, n-1$. The $n+1$ st operation transforms $I$ to $I^{\prime}$ and involves the identity inversion, i.e., $I$ inverts to $I$. The last $n$ operations by $S$ transform $I^{\prime}$ to $S^{n}\left(I^{\prime}\right)$. The inversions associated with these transformations are all in $I$ and are of the type $S^{n-j-1}\left(I^{\prime}\right)$ inverts to $S^{-(n-j)}(I)$. The latter $n$ inversions are thus inverses of the aforementioned $n$ inversions. Hence the resulting inversions associated with $S^{2 n+1}$ preserve infinitesimal lengths on $S^{-n}(I)$. The reflection and rotation components of $S^{2 n+1}$ clearly preserve infinitesimal lengths. Therefore $S^{-n}(I)$ is the isometric circle of $S^{2 n+1}$.

Part (iii) of the theorem is a consequence of the fact that an isometric circle may be written in the form $|z+d / c|=1 /|c|$.

We collect here some results on the inversion of one circle into another circle which will be needed subsequently. In the sequel, the circles $Q_{1}$ and $Q_{2}$ are always disjoint. If a circle $Q_{1}$ is inverted into a circle $Q_{2}$, we will designate the image circle by $Q_{12}$ and a corresponding 
subscript notation will be used for the radii $r$ and centers $q$ of the respective circles.

Let $Q_{1}$ and $Q_{2}$ be given by

$$
\begin{aligned}
& Q_{1}:\left|z-q_{1}\right|=r_{1}, \\
& Q_{2}:\left|z-q_{2}\right|=r_{2} .
\end{aligned}
$$

We denote by $l$ the line which passes through the centers of $Q_{1}, Q_{2}$ and we take the points $\alpha, \beta \in Q_{1} \cap l$. Suppose that $Q_{1}$ is inverted into $Q_{2}$ with $\alpha, \beta$ transforming into $\alpha_{i}, \beta_{i}$ respectively. Then

$$
\begin{aligned}
& \left|\alpha-q_{2}\right| \cdot\left|\alpha_{i}-q_{2}\right|=r_{2}^{2}, \\
& \left|\beta-q_{2}\right| \cdot\left|\beta_{i}-q_{2}\right|=r_{2}^{2} .
\end{aligned}
$$

We denote the distance between $q_{1}$ and $q_{2}$ by $e$ and obtain

$$
\begin{gathered}
r_{12}=\frac{r_{1} r_{2}^{2}}{\left|\alpha-q_{2}\right| \cdot\left|\beta-q_{2}\right|}=\frac{r_{1} r_{2}^{2}}{e^{2}-r_{1}^{2}}, \\
\left|q_{12}-q_{2}\right|=\frac{e r_{2}^{2}}{e^{2}-r_{1}^{2}} .
\end{gathered}
$$

LEMMA 13. Let $Q_{1}, Q_{2}$ be disjoint circles with centers $q_{1}, q_{2}$ and radii $r_{1}, r_{2}$ respectively. Then

(i) $r_{12}$ increases with increasing $r_{1}$ and fixed $e$ and also with decreasing $e$ and fixed $r_{1}$.

(ii) If $Q_{1}$ is enlarged to $Q_{1^{\prime}}$ in such a manner that $Q_{1} \subset Q_{1^{\prime}}$ and $Q_{1^{\prime}}$ is disjoint from $Q_{2}$, then $r_{1^{\prime} 2}>r_{12}$ and $r_{21^{\prime}}>r_{21}$.

(iii) $q_{12}$ lies on the line joining $q_{1}$ and $q_{2}$, and $\left|q_{2}-q_{12}\right|$ decreases with increasing $e$.

Proof. To prove (i) we note that because $Q_{1}$ and $Q_{2}$ are disjoint, $e>r_{1}$. The result then follows from equation (11).

For the proof of (ii), we denote the line passing through $q_{1}$ and $q_{2}$ by $l$. It is sufficient to consider the case in which the center $q_{1^{\prime}}$ lies on $l$ and one of the two points in $Q_{1} \cap l$ is fixed during the enlargement of $Q_{1}$. We use the first equation in (11) to find the total derivative with respect to $r_{1^{\prime}}$. We obtain the result that if $Q_{1^{\prime}}$ is inverted into $Q_{2}, d \mathrm{r}_{1^{\prime}} / d r_{1^{\prime}}>0$; and if $Q_{2}$ is inverted into $Q_{1^{\prime}}, d r_{21^{\prime}} / d r_{1^{\prime}}>0$. Because $r_{1^{\prime}}$ is steadily nondecreasing, we conclude that $r_{1^{\prime} 2}>r_{12}$ and $r_{21^{\prime}}>r_{21}$.

The first part of (iii) follows from elementary geometrical considerations of inversions. The second part of (iii) is obtained by differentiating, in equation (12), $\left|q_{2}-q_{12}\right|$ with respect to $e$ and noting that the derivative is negative.

3.5. Criterion for Vanishing Linear Measure. In the sequence 
of circles which bound the successive generations of mapped regions in the conformal mapping of $F_{S}^{*}$, the size of the circles is influenced only by the inversions associated with the elements of $G$ in the Schottky group. Suppose we enlarge any circle $\theta_{i} \in\left\{Q_{0}\right\}$ to $Q_{i^{\prime}}$ in such a manner that $Q_{i} \subset Q_{i^{\prime}}$ and $Q_{i^{\prime}}$ are disjoint from all other circles in $\left\{Q_{0}\right\}$. Then by repeated applications of Lemma 13 (i) and (ii) it follows that in the limit $m\left(E_{s}^{\prime}\right)$ for the new configuration will be greater than $m\left(E_{s}\right)$. Consequently, for establishing a criterion for the vanishing of $m\left(E_{s}\right)$, we may modify the configuration $\left\{Q_{0}\right\}$ to one in which all circles $Q_{i}$ are of equal unit size, subject to the conditions just mentioned. We will refer to this modified configuration of $\left\{Q_{0}\right\}$ as $\left\{Q_{0}\right\}_{A}$.

We consider the configuration $\left\{Q_{0}\right\}_{A}$. Let $e_{i}$ be the distance between the centers of the pair $Q_{i}$ and $Q_{i}^{\prime}(i=1,2, \cdots, p)$, and let ${ }_{i} d_{\text {, be the }}$ distance between the centers of two arbitrary circles $Q_{i}$ and $Q_{j} \in\left\{Q_{0}\right\}_{A}$. We denote by $e$ the minimum $e_{i}$ and by $d$ the minimum ${ }_{i} d_{j}$. If

$$
d \geqq e,
$$

we will say that $\left\{Q_{0}\right\}_{A}$ satisfies condition (B) and denote the configuration by $\left\{Q_{0}\right\}_{A B}$. The modified configuration $\left\{Q_{0}\right\}_{A B}$ will have a corresponding group of hyperbolic or loxodromic linear transformations $G^{\prime}$ which is associated with the Schottky group $G$ corresponding to $\left\{Q_{0}\right\}$. In the sequel, we will use the same notation for the circles in $\left\{Q_{0}\right\}_{A B}$ and for the generators of $G^{\prime}$ as used previously for those in $\left\{Q_{0}\right\}$ and in $\left\{S_{0}\right\}$ respectively.

THEOREM 9. Let G be a Schottky group with p generators. Suppose that there exists a configuration $\left\{Q_{0}\right\}_{A B}$ which is associated with G. Then the linear measure of the singular set of $G$ vanishes if

$$
p<\frac{e}{4}\left(e+\sqrt{e^{2}-4}\right) .
$$

Proof. Because of equations (3) and (6) in subsection 3.3 and because $Q_{i}$ and $Q_{i}$, are equal for all $i, Q_{i}$ and $Q_{i^{\prime}}$ are the isometric circles of the hyperbolic or loxodromic linear transformations $S_{i}$ and $S_{i}^{-1}$ respectively. Consequently, an arbitrary element of the group generated by $\left\{S_{0}\right\}$ is by Lemma 12 equivalent to a succession of compositions. Each of these compositions is an inversion in one of the circles $\left\{Q_{0}\right\}_{A B}$ followed by a reflection in the perpendicular bisector of the line joining the center of this circle to the center of its paired circle and a rotation about the center of some $Q_{i}$. We note that in the compositions, the size of the image circles is influenced only by the inversions.

Let $Q_{1}$ and $Q_{1}^{\prime}$ with centers at $q_{1}$ and $q_{1}^{\prime}$ respectively be that pair of circles in $\left\{Q_{0}\right\}_{A B}$ which has the minimum distance $e$ between their 
centers, and let $S_{1}$ be the corresponding generator. We may take $q_{1}$ at the origin and $q_{1}^{\prime}$ to be positive and real. Thus

$$
Q_{1}:|z|=1 ; \quad Q_{1}^{\prime}:\left|z-q_{1}^{\prime}\right|=1 .
$$

With this choice, we find from equation (6) that $a+d$ is real and $|a+d|>2$; hence $S_{1}$ is hyperbolic.

By hypothesis, the distance between $q_{1}$ and $q_{1}^{\prime}$ is smallest for the circles $Q_{1}$ and $Q_{1}^{\prime}$ in comparison with any other two circles in $\left\{Q_{0}\right\}_{A B}$; also, all of the circles in $\left\{Q_{0}\right\}_{A B}$ are equal. Consequently, we conclude from Lemma 13 (i) that the circle $S_{1}\left(Q_{1}^{\prime}\right) \subset Q_{1}^{\prime}$ has the maximal radius for all circles of the first generation. We denote by $q_{S_{1}}$ the center of $S_{1}\left(Q_{1}^{\prime}\right)$. By noting that $S_{1}$ is hyperbolic, it follows from Lemma 13 (iii) together with simple geometrical considerations that the distance between $q_{s_{1}}$ and $q_{1}$ is minimal in comparison with the distance between the center of any other circles $S_{i}\left(Q_{j}\right) \subset Q_{i}^{\prime}$ of the first generation and the center of any circle in $\left\{Q_{0}\right\}_{A B}$ exterior to $Q_{i}^{\prime}$. Consequently, if we apply Lemma 13 (i) again, we find that $S_{1}^{2}\left(Q_{1}^{\prime}\right) \subset Q_{1}^{\prime}$ has the maximal radius for all circles of the second generation.

Another application of Lemma 13 (iii) shows that the distance between $q_{s_{1}^{2}}$ and $q_{1}$ is minimal in comparison with the distance between the center of any other circle $S_{1} \circ S_{k}\left(Q_{j}\right) \subset Q_{1}^{\prime}$ of the second generation and the center of any circle in $\left\{Q_{0}\right\}_{A B}$ exterior to $Q_{1}^{\prime}$. Similarly we obtain a corresponding result for the $n$th generation. We conclude by induction that the circle $S_{1}^{n}\left(Q_{1}^{\prime}\right) \subset Q_{1}^{\prime}$ has the maximal radius for all circles of the $n$th generation for all $n$.

Let $r_{n}$ denote the radius of $S_{1}^{n}\left(Q_{1}^{\prime}\right) \subset Q_{1}^{\prime}$. We note that $S_{1}$ and $S_{1}^{-1}$ have the isometric circles $Q_{1}$ and $Q_{1}^{\prime}$ respectively. Consequently Theorem 8 (iii) is applicable and we obtain

$$
r_{n}=1 /|c|,
$$

where $c$ refers to the coefficient of the linear transformation corresponding to $S_{1}^{2 n+1}$. By utilizing this equation and equations (9), (13), (6), (7) and (8) and replacing $q_{1}^{\prime}$ by $e$, we obtain

$$
\begin{aligned}
r_{n} & =\left|\frac{\xi_{1}-\xi_{2}}{K^{\frac{2 n+1}{2}}-K^{\frac{-(2 n+1)}{2}}}\right| \\
& =\frac{\sqrt{e^{2}-4}}{\left.\mid \frac{-e+\sqrt{e^{2}-4}}{2}\right)^{2 n+1}-\left(\frac{-e+\sqrt{e^{2}-4}}{2}\right)^{-(2 n+1)}} \mid \\
& =\frac{\sqrt{e^{2}-4}}{\left(\frac{-e+\sqrt{e^{2}-4}}{2}\right)^{2 n+1}+\left(\frac{e+\sqrt{e^{2}-4}}{2}\right)^{2 n+1}} .
\end{aligned}
$$


The total number of circles in the $n$th generation is $2 p(2 p-1)^{n}$. We denote the total length of these circles by $L_{n}$. Then

$$
L_{n} \leqq \frac{4 \pi p(2 p-1)^{n} \sqrt{e^{2}-4}}{\left(\frac{-e+\sqrt{e^{2}-4}}{2}\right)^{2 n+1}+\left(\frac{e+\sqrt{e^{2}-4}}{2}\right)^{2 n+1}} .
$$

We find that

$$
\lim _{n \rightarrow \infty} L_{n}=0
$$

if

$$
p<\frac{\left(e+\sqrt{e^{2}-4}\right)^{2}+4}{8}=\frac{e\left(e+\sqrt{e^{2}-4}\right)}{4} .
$$

Because $m\left(E_{S}\right) \leqq \lim _{n \rightarrow \infty} L_{n}$, this is the required criterion.

Corollary. Suppose that the Schottky covering surface $F_{S}^{*}$ corresponds to a Schottky group $G$ with p generators. Let $G$ be associated with a configuration $\left\{Q_{0}\right\}_{A B}$ which satisfies Condition $C$ of Theorem 9. Then the boundary of the conformal equivalent of $F_{S}^{*}$ in the plane has zero linear measure.

Proof. By definition the boundary of the conformal equivalent of $F_{S}^{*}$ in the plane is the singular set of $G$. The conclusion then follows immediately from Theorem 9.

\section{Classification of Riemann Surfaces.}

4.1. Exhaustions AND HaRmonic Moduli. An are is analytic if it is the conformal image of a closed interval in the complex plane.

By virtue of the countability of a Riemann surface there always exists on such a surface an exhaustion which may be described as follows.

DEFINITION. A nested sequence $\left\{w_{n}\right\}$ of compact regions is an exhaustion of an open Riemann surface $W$ if

(i) $W_{n}$ is interior to $W_{n+1}$.

(ii) The boundary $\beta_{n}$ of $W_{n}$ consists of a finite number of closed disjoint piecewise analytic curves.

(iii) Each complement $W_{n}-W_{n-1}$ consists of a finite number of disjoint noncompact regions.

(iv) $\mathrm{U}_{n \rightarrow \infty} W_{n}=W$.

For every $n(n=0,1, \cdots)$, the complement $W_{n}-W_{n-1}$ consists of a finite number $k(n)$ of disjoint subregions $E_{n i}(i=1,2, \cdots, k(n))$ of finite genus. The boundary of $E_{n i}$ consists of two or more closed disjoint 
piecewise analytic curves which are subsets of $\beta_{n-1}$ and $\beta_{n}$. We denote the intersections of the boundary of $E_{n i}$ with $\beta_{n-1}$ and $\beta_{n}$, by $\beta_{n i}$ and $\beta_{n i}^{\prime}$ respectively. There exists on $E_{n i}$ a unique harmonic function $u_{n i}$ which is continuous on the closure of $E_{n i}$, vanishes on $\beta_{n i}$ and is constantly equal to unity on $\beta_{n i}^{\prime}$. The function $u_{n i}$ is called the harmonic measure of $\beta_{n i}^{\prime}$ with respect to $E_{n i}$.

If $E_{n i}$ is planar and $\beta_{n i}$ and $\beta_{n i}^{\prime}$ each consist of one component, then $E_{n i}$ is doubly connected. In this case, the function $U=e^{u_{n i}+i u^{*}} n i$ maps $E_{n i}$ conformally onto an annulus, where $u_{n i}^{*}$ represents the conjugate harmonic function of $u_{n i}$.

Let $E_{n i}(i=1,2, \cdots, k(n)<\infty, n=0,1, \cdots)$ be a collection of doubly-connected subregions of the open Riemann surface $W$, which may be represented as annuli and which satisfy the following conditions:

(i) Each annulus $E_{n i}$ is bounded by two closed, disjoint and piecewise analytic curves $\beta_{n i}$ and $\beta_{n i}^{\prime}$.

(ii) Any two of the annuli have no points in common.

(iii) The complementary set of $\bigcup_{i=1}^{k(n)} E_{n i}$ with respect to $W$ has precisely one compact component $W_{n}$.

(iv) $W_{n}$ is bounded by the $k(n)$ curves and contains the annuli $E_{n^{\prime} i}$ with $n^{\prime}<n$.

We define the harmonic modulus $\mu_{n i}$ of $E_{n i}$ as

$$
\mu_{n i}=2 \pi / \int_{\beta_{n i}} d u_{n i}^{*} \text {. }
$$

4.2. General Concept. The classification problem will be studied from the viewpoint of Sario [13] which classifies open Riemann surfaces according to their possession or nonpossession of a given property $P$ shared by all closed Riemann surfaces. If $W$ has the property $P$, we say that $W$ has a removable boundary with respect to $P$. Thus the behavior of the open surface with respect to $P$ is the same as if it were closed, that is, had no boundary. We will consider three properties shared by all closed Riemann surfaces, namely, they possess no $G, A D$ or $A B$ functions.

4.3. The Class $0_{G}$. The Green's function $g(z, \zeta)$ of a relatively compact Jordan region $R$ is defined as the unique harmonic function on $R$ which possesses the singularity $-\log |z-\zeta|$ at a point $\zeta \in R$ and which vanishes continuously on the boundary $\beta$ of $R$.

In order to generalize this definition to an arbitrary open Riemann surface, we will require the well-known Harnack's Principle which we state in the following form [2].

LEMma 14. Suppose that a family $\mathscr{Q}$ of harmonic functions on a 
Riemann surface $W$ satisfies the following condition.

To any $u_{i}$, $u_{j} \in \mathscr{U}$ there exists a $u_{k} \in \mathscr{U}$ with $u_{k} \geqq \max \left(u_{i}, u_{j}\right)$ on $W$. Then the function

$$
U(z)=\sup _{u_{i} \in \mathscr{U}} u_{i}(z)
$$

is either harmonic or constantly equal to $\infty$.

We consider an open Riemann surface $W$ and an exhaustion of the type described in subsection 4.1. If $W_{n}$ is one of the compact elements of the sequence $\left\{W_{n}\right\}$ in the exhaustion, its Green's function $g_{n}(z, \zeta)$ has the usual interpretation. By the maximum principle $g_{n}(z, \zeta)$ is a monotone increasing sequence of harmonic functions on $W$. Consequently by Harnack's principle, the sequence has a limiting function $g(z, \zeta)$ on $W$ which is either harmonic with the exclusion of the pole $-\log |z-\zeta|$ or else is identically infinite. In the first case we define $g(z, \zeta)$ to be Green's function for $W$ with a pole at $\zeta$. It can be shown that if the Green's function $g$ exists it is the smallest positive harmonic function with the singularity $-\log |z-\zeta|$. Also it satisfies the equality inf $g=0$. If a harmonic function with the same singularity as $g$ tends to 0 as $z$ approaches the boundary of $W$, then it is identical with $g$. We conclude that the Green's function is independent of the exhaustion.

Lemma 15. Mori [8]. Let $F_{h}^{*}$ be a homology covering surface of a closed Riemann surface $F$ and let $r\left[\Gamma\left(F_{h}^{*}\right)\right]$ be the rank of the group of cover transformations of $F_{h}^{*}$. Then $F_{h}^{*} \in 0_{G}$ if and only if $r\left[\Gamma\left(F_{n}^{*}\right)\right] \leqq 2$.

THEOREM 10. Let $F_{i}^{*}$ be a regular covering surface of a closed Riemann surface $F$ such that $F_{i}^{*}$ is weaker than the commutator covering surface of $F$, or equivalently

$$
T\left(F_{i}^{*}\right) \cong N_{i} \subset T(F), \quad N_{i} \supset N_{c}, \quad N_{i} / N_{c} \cong H_{i} \subset H(F) .
$$

Then $F_{i}^{*} \in 0_{G}$ if and only if

$$
r\left[\Gamma\left(F_{i}^{*}\right)\right]=r\left[\frac{T(F)}{N_{i}}\right]=r\left[\frac{H(F)}{H_{i}}\right]=r\left[\frac{T(F) / N_{c}}{N_{i} / N_{c}}\right] \leqq 2
$$

or equivalently

$$
2 p-2 \leqq r\left(H_{i}\right) \leqq 2 p
$$

Proof. To prove (i) we note that by Theorem $4, F_{i}^{*}$ is a homology covering surface. The conclusion then follows from Lemma 15 and Theorem 5 (i).

To prove (ii), we note that $F_{i}^{*}$ is a homology covering surface and $F$ is orientable. Consequently, Theorem 7 for the orientable case is 
applicable. We obtain

$$
r\left[\Gamma\left(F_{i}^{*}\right)\right]=2 p-r\left(H_{i}\right)
$$

in which

$$
0 \leqq r\left(H_{i}\right) \leqq r[H(F)]
$$

If $F_{i}^{*}$ satisfies (i),

$$
r\left[\Gamma\left(F_{i}^{*}\right)\right] \leqq 2
$$

Therefore,

$$
2 p-2 \leqq r\left(H_{i}\right) \leqq r[H(F)] .
$$

Conversely, we suppose that $F_{i}^{*}$ satisfies (ii). Then

$$
r\left[\Gamma\left(F_{i}^{*}\right)\right]=2 p-r\left(H_{i}\right) \leqq 2 p-(2 p-2)=2 .
$$

Hence, (i) and (ii) are equivalent.

4.4. THE Class $0_{A D}$. If $f(z)$ is an analytic function on a Riemann surface $W$, the Euclidean area of the image $W^{\prime}$ is given by the Dirichlet integral

$$
D(f)=\iint_{W}\left|f^{\prime}(z)\right|^{2} d x d y,
$$

where $z=x+i y$ is the local variable. It follows that the existence on $W$ of an $A D$ function implies the existence of a conformal equivalent of $W$ with finite Euclidean area. For simply-connected regions, the possibility of conformal equivalence with a finite or infinite disk is precisely the classical type problem. Hence the classification according to $0_{A D}$ is a generalization to arbitrary Riemann surfaces of this classical problem.

Lemma 16. Mori [8]. If $F_{h}^{*}$ is a homology covering surface of a closed Riemann surface, then $F_{h}^{*} \in 0_{A D}$.

THEOREM 11. If $F_{i}^{*}$ is a regular covering surface of a closed Riemann surface $F$ such that $F_{i}^{*}$ is weaker than the commutator covering surface of $F$, or equivalently,

$$
T\left(F_{i}^{*}\right) \cong N_{i} \subset T(F), \quad N_{i} \supset N_{c},
$$

then

$$
F_{i}^{*} \in 0_{A D}
$$


Proof By Theorem 4, $F_{i}^{*}$ is a homology covering surface. The result is then a consequence of Lemma 16.

4.5. The Class $0_{A B}$. If we consider an $A B$ function $f(z)$ defined in a region $W$, of the extended complex plane, which is complementary to a finite set of isolated points $\left\{p_{i}\right\}$, it is well known from the classical theory that the singularities $\left\{p_{i}\right\}$ can be removed by appropriately defining $f(z)$ at the points $p_{i}$. Painlevé [10] generalized this concept by investigating the analytic continuation of $A B$ functions across arbitrary point set boundaries of regions in the extended complex plane. This is the classical Painlevé's problem.

The connection of the classification according to $0_{A B}$ with Painlevé's problem is shown by the following lemma.

Lemma 17. [10], [1]. Suppose $E$ is a compact set in the extended plane and $W$ is its complement. Let $G$ be a relatively compact region in the plane with analytic boundary $\alpha$ and $E \subset G$. If $G_{0}=G-E$, then every $A B$ function, defined in $G_{0}$, possesses an analytic continuation to all of $G$, if and only if $W \in 0_{A B}$.

Proof. Suppose that $W \in 0_{A B}$. Let $F(z) \in A B$ be defined in $G_{0}$. By the compactness of $E$ we can enclose the points of $E$ in a finite number of piecewise analytic closed curves $\left\{C_{i}\right\}$. We apply Cauchy's integral formula to the region contained in $G$ but exterior to $\left\{C_{i}\right\}$. Then we can write

$$
f(z)=f_{1}(z)+f_{2}(z),
$$

where $f_{1}(z)$ is analytic in $G$, and $f_{2}(z)$ is analytic in the region exterior to $\left\{C_{i}\right\}$. We have for $f_{2}(z)$,

$$
\left|f_{2}(z)\right| \leqq M l / \rho,
$$

where $M$ is the supremum of $f(z), l$ is a finite length and $\rho>0$. Consequently $f_{2}(z)$ is an $A B$ function in $W$. Because $W \in 0_{A B}, f_{2}(z)$ is constant. Consequently $f_{1}(z)+$ constant is an analytic continuation of $f(z)$ across $E$.

Conversely, we suppose that the analytical continuation across $E$ is possible for every $A B$ function defined in $G_{0}$. If $f(z)$ is an $A B$ function on $W$, then the analytic continuation of $f(z)$ across $E$ is an $A B$ function in the extended plane. Therefore $f(z)$ must reduce to a constant. Hence we conclude that $W \in 0_{A B}$.

The lemma just proved shows that Painlevé's problem is the special case of the classification according to $0_{A B}$, where the surface is restricted to plane regions. 
The following lemma is implicit in the works of Painlevé [10].

Lemma 18. Let $E$ be a compact set in the extended plane and let $W$ be the complement of $E$. If the linear measure of $E$ is zero, then $W \in 0_{A B}$.

The following is a generalization of Lemma 18.

THEOREM 12. Let $W$ be an open Riemann surface with boundary $\beta$. Suppose that there exists a planar neighborhood $N$ of $\beta$ such that the relative boundary of $N$ is a single contour $\alpha$. If the boundary of the conformal equivalent of $N$ in the plane has zero linear measure, then $W \in 0_{A B}$.

Proof. $N$ is planar by hypothesis; therefore it can be mapped conformally onto a region $N^{\prime}$ of a disk $K:|z|<1$. In this mapping $\beta$ appears as a closed point set $E$ interior to $K$. The linear measure of $E$ vanishes by hypothesis; therefore by Lemma $18, W \in 0_{A B}$.

If $W$ is of finite genus $p$ with boundary $\beta$, then the postulated planar neighborhood of $\beta$ in Theorem 12 is assured. For in this case, we can find a compact region $W_{0} \subset W$, with genus $p$, bounded by a single contour $\alpha$, with $\alpha$ lying entirely in $W$. The complement $N=$ $W-W_{0}$ is then a planar neighborhood of $\beta$ and has a single contour $\alpha$ as its relative boundary. The following corollary is then an immediate consequence of Theorem 12 .

CoRollary. If $W$ is of finite genus and if the linear measure of $\beta$ vanishes under the conformal mapping of $N$ in the plane, then $W \in 0_{A B}$.

THeOREM 13. Let $F$ be a closed Riemann surface of finite genus p. Suppose that there exists for the Schottky covering surface $F_{S}^{*}$ of $F$ a modified configuration $\left\{Q_{0}\right\}_{A B}$, in the sense of subsection 3.5 such that $p<(e / 4)\left(e+\sqrt{e^{2}-4}\right)$. Then $F_{S}^{*} \in 0_{A B}$.

Proof. By the corollary to Theorem 9, the boundary of the conformal equivalent of $F_{S}^{*}$ in the plane has zero linear measure. We note that $F_{S}^{*}$ is an open Riemann surface of zero genus. The conclusion then follows from the corollary to Theorem 12.

We consider an open Riemann surface $W$ on which the domains of the homeomorphism $h_{i} \in\{h\}$ are denoted by $\Delta_{i}$. Let $\lambda(z)$ be a continuous and positive (except for isolated points) function on each domain $\Delta_{i}$ of $W$. If two domains $\Delta_{j}$ and $\Delta_{k}$ overlap, let $\lambda(z)$ satisfy the covariance relation 


$$
\lambda\left(z_{j}\right)=\lambda\left(z_{k}\right)\left|\frac{d z_{k}}{d z_{j}}\right|
$$

at corresponding points $z_{j}$ and $z_{k}$ in $\Delta_{j} \cap \Delta_{k}$. We further require that all points in $W$ have an infinite distance from the ideal boundary of $W$. We say that the differential

$$
d s=\lambda(z)|d z|
$$

defines a conformal metric on $W$, if it satisfies all the conditions just indicated.

Suppose that a conformal metric is defined on $W$. We fix a point 0 in $W$ and let $D_{\rho}$ be the domain formed by those points whose distance from 0 is less than $\rho$, where $0<\rho<\infty$. For $\rho<\infty$, we assume that the domains are compact and that they generate $W$ as $\rho \rightarrow \infty$. Each domain $D_{\rho}$ is bounded by $\beta_{\rho}$, where $\beta_{\rho}$ consists of a finite number $k(\rho)$ of closed disjoint piecewise analytic curves, $\beta_{\rho 1}, \beta_{\rho 2}, \cdots, \beta_{\rho[k(\rho)]}$. Let

$$
\begin{array}{rlrl}
l_{i} & =\int_{\beta \rho_{i}} d s, & i=1,2, \cdots, k(\rho), \\
\Lambda(\rho) & =\max _{i} \int_{\beta \rho_{i}} d s, \\
K(N) & =\max _{\rho^{\prime} \leqq \rho} k\left(\rho^{\prime}\right) .
\end{array}
$$

Then we have

Lemma 19. (Pfluger [11]). If

$$
\varlimsup_{N \rightarrow \infty}\left[4 \pi \int_{0}^{N} \frac{d \rho}{\Lambda(\rho)}-\log K(N)\right]=\infty
$$

on $W$, then $W \in 0_{A B}$.

In [8], Mori states without proof a modification of Lemma 19 which does not involve the assumption of a conformal metric on the surface. For the modified version of the lemma, we assume an exhaustion of $W$ and obtain as in subsection 4.1 the corresponding collection of annuli $\left\{E_{n i}\right\}$. We set

$$
\begin{aligned}
\mu_{n}=\min _{i} \mu_{n i} & =2 \pi / \int_{\beta_{n i}} d u_{n i}^{*}, \\
K(N) & =\max _{n \leqq N} k(n) .
\end{aligned}
$$

Then we prove

LEMMA 20. If

$$
\varlimsup_{N \rightarrow \infty}\left\{\sum_{n=j}^{N} \mu_{n}-\frac{1}{2} \log K(N)\right\}=\infty,
$$


then

$$
W \in 0_{A B}
$$

Proof. We consider the postulated exhaustion of $W$ and the corresponding annuli $\left\{E_{n i}\right\}$. Let $E_{n i}$ be one such annulus which is bounded by $\beta_{n i}$ and $\beta_{n i}^{*}$ and let $u_{n i}(z)$ be the harmonic measure of $\beta_{n i}^{*}$ with respect to $E_{n i}$. By the maximum principle, $0<u_{n i}(z)<1$ in $E_{n i}$. We define the function $u_{n i}(z)$ to be the distance of the point $z$ from $\beta_{n i}$. Then the function $\left|\operatorname{grad} u_{n i}(z)\right|$ defines a conformal metric on the annulus $E_{n i}$, for which

$$
d s=\left|\operatorname{grad} u_{n i}(z)\right||d z| .
$$

Let $\beta_{\rho_{i}}$ denote the set of points on $E_{n i}$ which have the distance $\rho$ from $\beta_{n i}$. Then

$$
l_{i}=\int_{\beta_{\beta_{i}}} d s=\int_{\beta_{\rho_{i}}} \frac{\partial u_{n i}}{\partial n}|d z|=\int_{\beta_{\rho_{i}}} d u_{n i}^{*}=\frac{2 \pi}{\mu_{n i}}
$$

where $\partial n$ is normal to $d s$.

The result then follows from Lemma 19.

In [8], Mori utilized Lemma 20 to prove

Lemma 21. Let $F_{h}^{*}$ be a homology covering surface of a closed Riemann surface $F$. Suppose that the group of cover transformation $\Gamma\left(F_{h}^{*}\right)$ has the system of $2 p$ generators $C_{2 i-1}, C_{2 i}(i=1,2, \cdots, p)$. If there exists for each $i$ a relation of the form

$$
\gamma_{2 i-1} C_{2 i-1}+\gamma_{2 i} C_{2 i}=0
$$

where $\gamma_{2 i-1}$ and $\gamma_{2 i}$ are integers and do not vanish simultaneously, then $F_{h}^{*} \in 0_{A B}$.

Let $F$ be a closed Riemann surface of genus $p$. Suppose that $F$ is cut along $p$ disjoint nondividing cycles to produce a planar surface $F_{0}^{\prime}$. Following Royden [12], we shall refer to a regular covering surface $F^{*}$ of $F$ as a covering surface of type $S$, if it consists of a finite or infinite number of copies of $F_{0}^{\prime}$.

Corollary. [8]. A homology covering surface $F_{n}^{*}$ of type $S$ of a closed Riemann surface $F$ is in $0_{A B}$.

Proof. Let the $2 p$ nondividing cycles $C_{2 i-1}, C_{2 i}(i=1,2, \cdots, p)$ correspond to the $2 p$ generators of $\Gamma\left(F_{n}^{*}\right)$. If we cut $F$ along the nondividing cycles $C_{2 i-1}(i=1,2, \cdots, p)$, then the cycles $C_{2 i-1}$ correspond to the identity element in $\Gamma\left(F_{h}^{*}\right)$. Hence we may take $\gamma_{2 i-1}=1$ and $\gamma_{2 i}=0$ and obtain 


$$
\gamma_{2 i-1} C_{2 i-1}+\gamma_{2 i} C_{2 i}=0, \quad(i=1,2, \cdots, p) .
$$

The conclusion then follows from Lemma 21.

Theorem 14. Let $F_{i}^{*}$ be a regular covering surface of a closed Riemann surface $F$ of genus $p$ such that $F_{i}^{*}$ is weaker than the commutator covering surface of $F$, or equivalently,

$$
T\left(F_{i}^{*}\right) \cong N_{i} \subset T(F), \quad N_{i} \supset N_{c}, \quad\left(N_{i} / N_{c}\right) \cong H_{i} \subset H(F) .
$$

Suppose that

(i) $\Gamma\left(F_{i}^{*}\right)$ has the $2 p$ generators $C_{2 i-1}, C_{2 i}(i=1,2, \cdots, p)$ such that $C_{2 i-1}, C_{2 i}$ correspond respectively to $a_{2 i-1}, a_{2 i}$ under the isomorphisms of Theorem 5 (i). If there exists for each $i=1,2, \cdots, p$ a relation of the form

$$
\gamma_{2 i-1} a_{2 i-1}+\gamma_{2 i} a_{2 i}=0
$$

where $\gamma_{2 i-1}$ and $\gamma_{2 i}$ are integers and do not vanish simultaneously, and $a_{2 i-1}, a_{2 i}(i=1,2, \cdots, p)$ refer to the $2 p$ generators of the Abelian groups

$$
\frac{H(F)}{H_{i}}, \frac{T(F)}{N_{i}} \text { and } \frac{T(F) / N_{c}}{N_{i} / N_{c}}
$$

or

(ii) $F_{i}^{*}$ is of type $S$, then $F_{i}^{*} \in 0_{A B}$.

Proof. By Theorem $4, F_{i}^{*}$ is a homology covering surface. The conclusion then follows from Lemma 21 and its corollary in conjunction with Theorem 5 (i).

\section{REFERENCES}

1. Lars Ahlfors and Arne Beurling, Conformal invariants and function-theoretic null-sets, Acta Math., 83 (1950), 101-129.

2. Lars Ahlfors and Leo Sario, Riemann Surfaces, Princeton University Press, Princeton 1960.

3. R. Courant, Geometrische Funktionentheorie. Hurwitz-Courant: Funktionentheorie, Springer, Berlin, (1925), 476-484.

4. Lester R. Ford, Automorphic Functions, McGraw-Hill, New York, (1929), 23.

5. B. V. Kerékjárto, Vorlesungen Uber Topologie, Springer, Berlin, (1923), 182-184.

6. A. G. Kurosh, The Theory of Groups, Chelsea, New York, 1 (1955), 71-74.

7. - The Theory of Groups, Chelsea, New York, 2 (1955), 92.

8. A. Mori, A note on unramified Abelian covering surfaces of a closed Riemann surface, J. Math. Soc. Japan, 6 (1954), 162-176.

9. Rolf Nevanlinna, Uniformisierung, Die Grundlehren der Mathematischen Wissenschaften in Einzeldarstellungen mit besonderer Berücksichtigung der Anwendungsgebiete, Bd. LXIV, Springer-Verlag, Berlin-Gottingen-Heidelberg, (1953), 64-83. 
10. P. Painlevé, Sur les lignes singulières des fonctions analytiques, Ann. Fac. Sci. Toulouse, 2 (1888).

11. Albert Pfluger, Sur l'existence de fonctions non constantes, analytiques, uniformes et bornées sur une surface de Riemann Ouverte, C. R. Acad. Sci. Paris, 230 (1950), 166-168. 12. H. L. Royden, Harmonic functions on open Riemann surfaces, Trans. Amer. Math. Soc., 73 (1952), 40-94.

13. Leo Sario, Uber Riemannsche Flachen mit hebbarem Rand, Ann. Acad. Sci. Fenn. Ser. A. I. Math.-Phys., 50 (1948).

14. _- Modular criteria on Riemann surfaces, Duke Math. J., 20 (1953), 279-286.

15. F. Schottky, Ueber eine specielle Function, welche bei einer bestimmten linearen Transformation ihres Argumentes unverändert bleibt, Crelle's J., 101 (1887), 227.

16. H. Seifert and W. Threlfall, Lehrbuch der Topologie, Teubner, Leipzig und Berlin, 1934.

17. H. Weyl, Die Idee der Riemannschen Flache, Teubner, Leipzig und Berlin, 1913.

Fresno State College 



\section{PACIFIC JOURNAL OF MATHEMATICS}

\section{EDITORS}

David GILbarg

Stanford University

Stanford, California

\section{F. H. BRowneLL}

University of Washington

Seattle 5, Washington

\section{A. L. Whiteman}

University of Southern California Los Angeles 7, California

\section{J. PAIGe}

University of California

Los Angeles 24, California

\section{ASSOCIATE EDITORS}
E. F. BECKENBACH
T. M. CHERRY
D. DERRY

\author{
E. HEWITT \\ A. HORN \\ L. NACHBIN
}

\author{
M. OHTSUKA \\ H. L. ROYDEN \\ M. M. SCHIFFER
}

E. SPANIER

E. G. STRAUS

F. WOLF

\section{SUPPORTING INSTITUTIONS}

\author{
UNIVERSITY OF BRITISH COLUMBIA \\ CALIFORNIA INSTITUTE OF TECHNOLOGY \\ UNIVERSITY OF CALIFORNIA \\ MONTANA STATE UNIVERSITY \\ UNIVERSITY OF NEVADA \\ NEW MEXICO STATE UNIVERSITY \\ OREGON STATE COLLEGE \\ UNIVERSITY OF OREGON \\ OSAKA UNIVERSITY \\ UNIVERSITY OF SOUTHERN CALIFORNIA
}

\author{
STANFORD UNIVERSITY \\ UNIVERSITY OF TOKYO \\ UNIVERSITY OF UTAH \\ WASHINGTON STATE COLLEGE \\ UNIVERSITY OF WASHINGTON \\ AMERICAN MATHEMATICAL SOCIETY \\ CALIFORNIA RESEARCH CORPORATION \\ HUGHES AIRCRAFT COMPANY \\ SPACE TECHNOLOGY LABORATORIES \\ NAVAL ORDNANCE TEST STATION
}

\footnotetext{
Mathematical papers intended for publication in the Pacific Journal of Mathematics should be typewritten (double spaced), and the author should keep a complete copy. Manuscripts may be sent to any one of the four editors. All other communications to the editors should be addressed to the managing editor, L. J. Paige at the University of California, Los Angeles 24, California.

50 reprints per author of each article are furnished free of charge; additional copies may be obtained at cost in multiples of 50 .
}

The Pacific Journal of Mathematics is published quarterly, in March, June, September, and December. The price per volume (4 numbers) is $\$ 12.00$; single issues, $\$ 3.50$. Back numbers are available. Special price to individual faculty members of supporting institutions and to individual members of the American Mathematical Society: $\$ 4.00$ per volume; single issues, $\$ 1.25$.

Subscriptions, orders for back numbers, and changes of address should be sent to Pacific Journal of Mathematics, 2120 Oxford Street, Berkeley 4, California.

Printed at Kokusai Bunken Insatsusha (International Academic Printing Co., Ltd.), No. 6, 2-chome, Fujimi-cho, Chiyoda-ku, Tokyo, Japan.

PUBLISHED BY PACIFIC JOURNAL OF MATHEMATICS, A NON-PROFIT CORPORATION

The Supporting Institutions listed above contribute to the cost of publication of this Journal, but they are not owners or publishers and have no responsibility for its content or policies. 


\section{Pacific Journal of Mathematics}

\section{Vol. 10, No. $4 \quad$ December, 1960}

M. Altman, An optimum cubically convergent iterative method of inverting a linear bounded operator in Hilbert space . . . . . . . . . . . . . . . . . . . . . . . . . . 1107

Nesmith Cornett Ankeny, Criterion for rth power residuacity ................. 1115

Julius Rubin Blum and David Lee Hanson, On invariant probability measures I . . . . . 1125

Frank Featherstone Bonsall, Positive operators compact in an auxiliary topology ..... 1131

Billy Joe Boyer, Summability of derived conjugate series . . . . . . . . . . . . . . . . 1139

Delmar L. Boyer, A note on a problem of Fuchs . . . . . . . . . . . . . . . . . 1147

Hans-Joachim Bremermann, The envelopes of holomorphy of tube domains in infinite

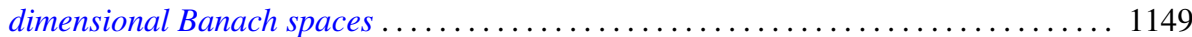

Andrew Michael Bruckner, Minimal superadditive extensions of superadditive

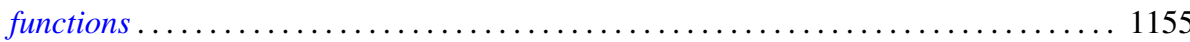

Billy Finney Bryant, On expansive homeomorphisms .................... 1163

Jean W. Butler, On complete and independent sets of operations in finite algebras . . . . . 1169

Lucien Le Cam, An approximation theorem for the Poisson binomial distribution ...... 1181

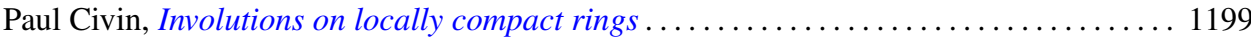

Earl A. Coddington, Normal extensions of formally normal operators . . . . . . . . . 1203

Jacob Feldman, Some classes of equivalent Gaussian processes on an interval ........ 1211

Shaul Foguel, Weak and strong convergence for Markov processes . . . . . . . . . . . 1221

Martin Fox, Some zero sum two-person games with moves in the unit interval ........ 1235

Robert Pertsch Gilbert, Singularities of three-dimensional harmonic functions . . . . . . . 1243

Branko Grünbaum, Partitions of mass-distributions and of convex bodies by

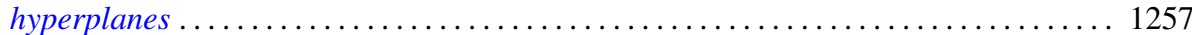

Sidney Morris Harmon, Regular covering surfaces of Riemann surfaces ........... 1263

Edwin Hewitt and Herbert S. Zuckerman, The multiplicative semigroup of integers

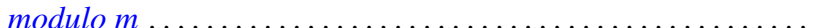

Paul Daniel Hill, Relation of a direct limit group to associated vector groups . ......... 1309

Calvin Virgil Holmes, Commutator groups of monomial groups . .

James Fredrik Jakobsen and W. R. Utz, The non-existence of expansive homeomorphisms

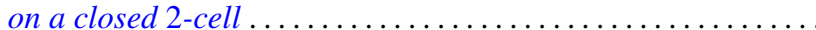

John William Jewett, Multiplication on classes of pseudo-analytic functions . . . . . . . 1323

Helmut Klingen, Analytic automorphisms of bounded symmetric complex domains . . . . 1327

Robert Jacob Koch, Ordered semigroups in partially ordered semigroups . . . . . . . . 1333

Marvin David Marcus and N. A. Khan, On a commutator result of Taussky and

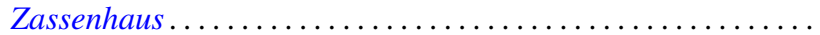

John Glen Marica and Steve Jerome Bryant, Unary algebras......

Edward Peter Merkes and W. T. Scott, On univalence of a continued fraction . . . . . . . 1361

Shu-Teh Chen Moy, Asymptotic properties of derivatives of stationary measures . . . . . 1371

John William Neuberger, Concerning boundary value problems . . . . . . . . . . . 1385

Edward C. Posner, Integral closure of differential rings . . . . . . . . . . . . . . . . . 1393

Marian Reichaw-Reichbach, Some theorems on mappings onto . . . . . . . . . . . . . 1397

Marvin Rosenblum and Harold Widom, Two extremal problems . . . . . . . . . . . . . . . . 1409

Morton Lincoln Slater and Herbert S. Wilf, A class of linear differential-difference

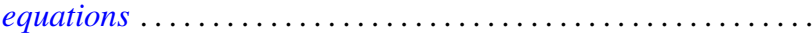

Charles Robson Storey, Jr., The structure of threads . . . . . . . . . . . . . . . . . . 1429

J. François Treves, An estimate for differential polynomials in $\partial / \partial z_{1},, \cdots, \partial / \partial z_{-} n \ldots \ldots 1447$

J. D. Weston, On the representation of operators by convolutions integrals . . . . . . . . 1453

James Victor Whittaker, Normal subgroups of some homeomorphism groups ......... 1469 